\title{
LQR Control of Wind Excited Benchmark Building Using Variable Stiffness Tuned Mass Damper
}

\author{
S. N. Deshmukh and N. K. Chandiramani \\ Department of Civil Engineering, Indian Institute of Technology Bombay, Mumbai 400076, India \\ Correspondence should be addressed to N. K. Chandiramani; naresh@civil.iitb.ac.in
}

Received 6 September 2012; Accepted 6 October 2013; Published 8 April 2014

Academic Editor: Jeong-Hoi Koo

Copyright (C) 2014 S. N. Deshmukh and N. K. Chandiramani. This is an open access article distributed under the Creative Commons Attribution License, which permits unrestricted use, distribution, and reproduction in any medium, provided the original work is properly cited.

\begin{abstract}
LQR control of wind induced motion of a benchmark building is considered. The building is fitted with a semiactive variable stiffness tuned mass damper adapted from the literature. The nominal stiffness of the device corresponds to the fundamental frequency of the building and is included in the system matrix. This results in a linear time-invariant system, for which the desired control force is computed using LQR control. The control force thus computed is then realized by varying the device stiffness around its nominal value by using a simple control law. A nonlinear static analysis is performed in order to establish the range of linearity, in terms of the device (configuration) angle, for which the control law is valid. Results are obtained for the cases of zero and nonzero structural stiffness variation. The performance criteria evaluated show that the present method provides displacement control that is comparable with that of two existing controllers. The acceleration control, while not as good as that obtained with the existing active controller, is comparable or better than that obtained with the existing semiactive controller. By using substantially less power as well as control force, the present control yields comparable displacement control and reasonable acceleration control.
\end{abstract}

\section{Introduction}

Active control devices, such as the Active Tuned Mass Damper (ATMD), require substantial input power and could also destabilize the system if the controller is improperly designed. On the other hand, passive control devices are less effective in the presence of stochastic disturbances and/or structural property variations. Semiactive control devices do not possess these disadvantages and thus appear to be sound alternatives to active and passive devices [1-4]. Such devices provide control forces by varying their mechanical properties, based on feedback. The variable stiffness damper is a semiactive device with good potential for controlling wind/earthquake generated response. Kobori et al. [5] and Nasu et al. [6] considered an active variable stiffness (AVS) system, comprising an on-off type two ended hydraulic damper, to make the structure nonresonant during an earthquake. Nemir et al. [7] considered a variable stiffness bracing and obtained rapid dissipation by way of energy redistribution to higher modes. Such AVS systems, while effective, cause abrupt switching of stiffness. Yang et al. [8] proposed a sliding mode controller for an AVS system. A resetting control algorithm, involving the release of potential energy of the device followed by a quick resetting of the device to its full-stiffness state, was considered by Yang et al. [9]. Yang et al. [2] proposed a 76-storey building in Melbourne as a benchmark structure for evaluating algorithms for wind induced response control. Results using LQG control with an ATMD were obtained in their study.

Nagarajaiah [10] developed a semiactive variable stiffness (SAVS) device and studied its performance using a scaled model. The SAVS-TMD has been shown to be effective for structures that are subjected to force/base excitation [11]. Varadarajan and Nagarajaiah [12,13] studied the wind response control of the benchmark building [2]. They used Empirical Mode Decomposition-Hilbert Transform method [12] and Short Time Fourier Transform (STFT) method [13] in order to track the dominant response frequency. The SAVS-TMD was then tuned to this frequency. Wu and Yang [14] studied the performance of Linear Quadratic Gaussian control (LQG), $H_{\infty}$ control, and continuous sliding mode control applied to an active mass driver, for acceleration 


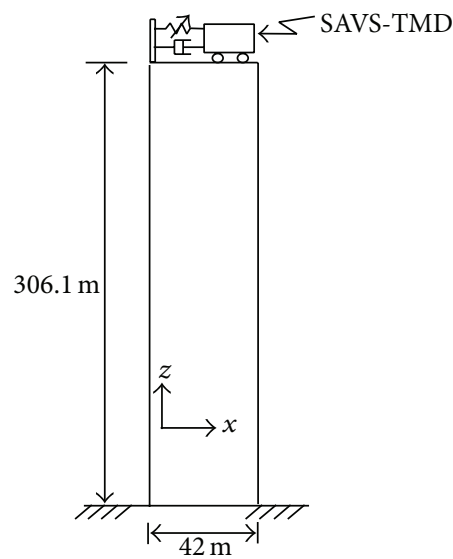

(a)

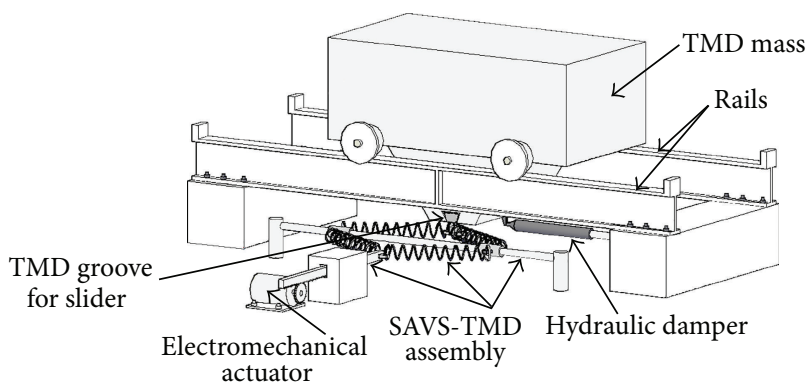

(b)

Figure 1: (a) Benchmark building with SAVS-TMD. (b) Realization of SAVS-TMD inspired by [13].

reduction of the wind excited Nanjing tower. Using a variable stiffness TMD, Collins et al. [15] considered bangbang control combined with semiactive control. However, they did not consider the actuator dynamics. Semiactive controller designs using other devices are also available. For magnetorheological devices, Yuen et al. [16] used reliability based robust linear control with a clipped control law, and Karamodin and Kazemi [17] used LQG control and a semiactive neural controller with acceleration/velocity feedback. Sohn et al. [18] studied the semiactive control of a suspension system, by estimating the road profile using the extended least squares method and then applying LQG control. Gaul et al. [19] studied the control of a truss with semiactive friction joints. They used two methods, that is, one with a controller for each joint and another with a single clipped-optimal controller.

In the present study, the SAVS-TMD of [12, 13] is deployed in order to control the wind excited benchmark building [2] by using a Linear Quadratic Regulator (LQR) controller. The nominal stiffness of the device, corresponding to the fundamental frequency of the structure, is included in the system matrix. This results in a linear time invariant system, for which algorithms suitable for real-time control applications can be employed. One such algorithm is LQR control wherein gains are computed offline, thus making it suitable for real-time control. The desired control force is computed using LQR control and then realized by changing the stiffness of the device within limits specified around the nominal stiffness. This is done using a simple control law which changes the configuration of the device-within specified limits-by means of an electromechanical actuator. The dynamics of the actuator are excluded from this study. A nonlinear static analysis is performed in order to obtain the operational range of the device configuration angle. This ensures a linear force-displacement behavior for the device, and hence validity of the control law. In order to assess robustness of control, the controller thus designed is implemented on the structure having $\pm 15 \%$ stiffness variation [2]. The goals of the paper are (i) implementation of STLC, that is, the SAVS-TMD device with LQR control, with a simple control law that is valid within the operational range of the device, (ii) comparing the performance of STLC with that of the controller in [2], which is based on ATMD with LQG control (ALC), and the controller in [13] which is based on SAVS-TMD with STFT control (STSC). This is done for all cases of structural stiffness variation, in order to assess control robustness. In contrast to STLC where gains are computed offline (i.e., computed only once), STFT involves a time-varying system with online computations for real-time frequency tracking during its control law implementation, so as to tune the device to the tracked frequency. This involves intensive online computations which increase the controlloop time and thus renders STFT less suitable for real-time control. On the other hand, ALC, being an active method, requires substantially more power and control force than STLC. Thus, the present study provides a new power-efficient controller design for the benchmark problem, that is, one which is suitable for real-time control and which can be readily extended to output-based feedback control in order to further decrease the loop time.

\section{Semiactive Variable Stiffness TMD}

The SAVS-TMD is fitted at the top of the 76-storey benchmark building [2], as shown in Figure 1(a). The device comprises a rhombus of four springs, each having stiffness $k_{e}$ and unstretched length $L_{e}$. The springs are pin connected at sliding joints (Figures 1(b) and 2). The masses of sliders and springs, and the effect of friction, are neglected. Joint- 3 and joint- 4 slide along a horizontal guide-rail fixed on the floor. Joint-2 slides along the $y$-directed groove which is present at the bottom of the TMD mass. The TMD mass moves along the $x$-directed rails that are fixed on the floor. By using a controlled actuator, joint- 1 can be made to move along a $y$-directed guide that is fixed on the floor. This causes the stiffness of the device to vary, due to variation in the device configuration angle $\theta$. 


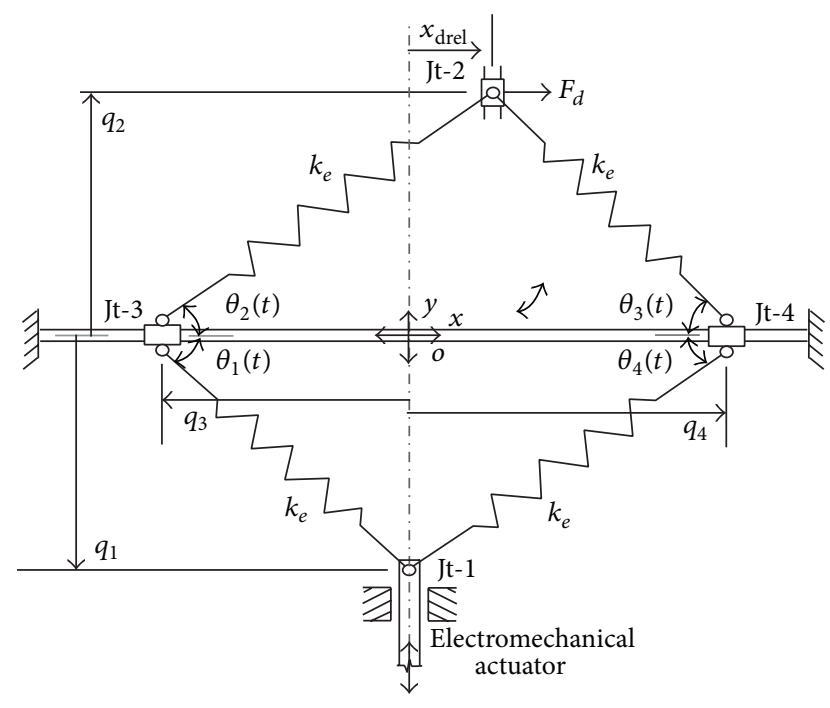

FIgURE 2: Schematic of SAVS with TMD displaced.

The coordinates of joints 1,3 , and 4 are denoted $q_{1}, q_{3}$, and $q_{4}$, respectively. The $x$ and $y$ coordinates of joint-2 are denoted $x_{\text {drel }}$ and $q_{2}$, respectively, with $x_{\text {drel }}$ being the TMD displacement measured relative to the top storey (Figure 2). The joint coordinates are measured as per directions shown in Figure 2. The TMD displacement results in force $F_{d}$ at joint-2, measured positive rightward. Equilibrium of joints 2, 3, and 4 yields

$$
\begin{gathered}
F_{23} \sin \theta_{2}+F_{24} \sin \theta_{3}=0 ; \\
F_{23} \cos \theta_{2}+F_{13} \cos \theta_{1}=0 ; \\
F_{24} \cos \theta_{3}+F_{14} \cos \theta_{4}=0 ; \\
F_{23} \cos \theta_{2}-F_{24} \cos \theta_{3}-F_{d}=0,
\end{gathered}
$$

where

$$
\begin{gathered}
F_{13}=k_{e}\left(\sqrt{q_{3}^{2}+q_{1}^{2}}-L_{e}\right) ; \\
F_{23}=k_{e}\left(\sqrt{\left(q_{3}+x_{\mathrm{drel}}\right)^{2}+q_{2}^{2}}-L_{e}\right) ; \\
F_{14}=k_{e}\left(\sqrt{q_{4}^{2}+q_{1}^{2}}-L_{e}\right) ; \\
F_{24}=k_{e}\left(\sqrt{\left(q_{4}-x_{\mathrm{drel}}\right)^{2}+q_{2}^{2}}-L_{e}\right)
\end{gathered}
$$

are the spring forces. Here $\theta_{1}, \ldots, \theta_{4}$ are expressed in terms of joint coordinates. For example, $\cos \theta_{2}=\mathrm{C}_{3}+$ $\left.x_{\text {drel }}\right) /\left(\sqrt{\left(q_{3}+x_{\text {drel }}\right)^{2}+q_{2}^{2}}\right)$.

For $q_{1}$ held fixed and $x_{\text {drel }}=0$, the equilibrium configuration angles are equal and denoted as $\theta$; that is, $\theta_{1}=$ $\theta_{2}=\theta_{3}=\theta_{4}=\theta=\sin ^{-1}\left(q_{1} / L_{e}\right)$. Thus, $\theta$, or equivalently $q_{1}$, represents the device configuration. Choosing device configuration $q_{1}$ (which is equivalent to choosing $\theta$ ), $q_{2}, q_{3}$, $q_{4}$, and $F_{d}$ are obtained for various TMD displacements,

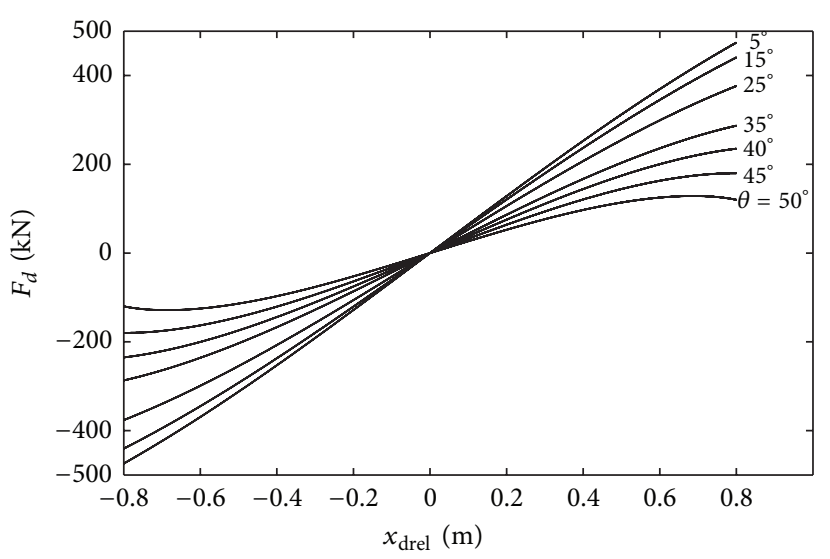

Figure 3: $F_{d}$ versus $x_{\text {drel }}$ for various configurations $\theta$.

$x_{\text {drel }}$, by solving (1) using MATLAB $f$ solve. Thus, the forcedisplacement behavior of the SAVS-TMD, that is, $F_{d}$ versus $x_{\text {drel }}$, is obtained as shown in Figure 3. Here, $L_{e}=2 \mathrm{~m}$, $k_{e}=650 \mathrm{kN} / \mathrm{m}$, and various device configurations $\theta$, such that $5^{\circ} \leq \theta \leq 50^{\circ}$, are considered. The behavior is almost linear up to $\theta=25^{\circ}$, beyond which the linearity holds within a range of $x_{\text {drel }}$. This range reduces as the device configuration $\theta$ (or $q_{1}$ ) increases. The nonlinearity becomes significant as the device configuration angle increases. The nonlinear behavior begins, for example, at a TMD displacement of around $0.55 \mathrm{~m}$ for $\theta=40^{\circ}$ and at a TMD displacement of around $0.45 \mathrm{~m}$ for $\theta=45^{\circ}$. Thus, for large TMD-displacement, the device behaves nonlinearly when the device configuration angle is also large.

Assuming that $x_{\mathrm{drel}} \ll L_{e}$, that is, the TMD displacement is small relative to the unstretched spring length, the model of $[12,13]$ can be used. This model implies a linear forcedisplacement relation for a given configuration $\theta$, with device stiffness given as

$$
k_{d}(t)=k_{e} \cos ^{2} \theta(t) .
$$

Here, $k_{d}(t)$ is the stiffness provided by the device for a given configuration $\theta$. Based on the force-displacement behavior shown in Figure 3, the maximum configuration angle is restricted to $\theta_{\max }=41^{\circ}$ (i.e., open position) in order to maintain device linearity. This corresponds to around 10\% nonlinearity at $x_{\text {drel }}=0.68$ (Figure 3 ). Further, the minimum configuration angle is restricted to $\theta_{\min }=7^{\circ}$ (i.e., closed position) due to mechanical constraints during flattening of the rhombus. The nominal configuration angle of the device is set as $\theta_{n}=28.2^{\circ}$ in order that, in this configuration, the device is tuned to the fundamental frequency of the structure. Depending on the control force required, the device stiffness is increased/decreased by varying the device configuration, $\theta$, about $\theta_{n}$, such that $\theta_{\min } \leq \theta \leq \theta_{\max }$. With the linear forcedisplacement relation of (3) used in the controller design, the RMS value of TMD displacement is obtained as less than $26 \mathrm{~cm}$, as seen from the controlled responses in Table 1 . When both the control-force required and the TMD-displacement are large and have opposite signs, the device angle $\theta$ required is large and the force-displacement relation is nonlinear 
TABLE 1: Performance criteria: STLC compared with ALC and STSC, for $0 \%$ and $\pm 15 \%$ structural stiffness variations.

\begin{tabular}{|c|c|c|c|c|c|c|c|}
\hline \multicolumn{4}{|c|}{ RMS response } & \multicolumn{4}{|c|}{ Peak response } \\
\hline Criterion & $0 \%$ & $15 \%$ & $-15 \%$ & Criterion & $0 \%$ & $15 \%$ & $-15 \%$ \\
\hline \multirow{3}{*}{$J_{1}$} & 0.44 & 0.45 & 0.45 & \multirow{3}{*}{$J_{7}$} & 0.44 & 0.51 & 0.52 \\
\hline & $\{0.37\}$ & $\{0.37\}$ & $\{0.39\}$ & & $\{0.38\}$ & $\{0.41\}$ & $\{0.49\}$ \\
\hline & {$[0.47]$} & {$[0.46]$} & {$[0.50]$} & & {$[0.45]$} & {$[0.50]$} & {$[0.57]$} \\
\hline \multirow{3}{*}{$J_{2}$} & 0.44 & 0.45 & 0.45 & \multirow{3}{*}{$J_{8}$} & 0.45 & 0.50 & 0.55 \\
\hline & $\{0.42\}$ & $\{0.41\}$ & $\{0.44\}$ & & $\{0.43\}$ & $\{0.44\}$ & $\{0.54\}$ \\
\hline & {$[0.46]$} & {$[0.45]$} & {$[0.50]$} & & {$[0.46]$} & {$[0.47]$} & {$[0.57]$} \\
\hline \multirow{3}{*}{$J_{3}$} & 0.58 & 0.50 & 0.70 & \multirow{3}{*}{$J_{9}$} & 0.71 & 0.64 & 0.81 \\
\hline & $\{0.58\}$ & $\{0.49\}$ & $\{0.71\}$ & & $\{0.72\}$ & $\{0.61\}$ & $\{0.77\}$ \\
\hline & {$[0.60]$} & {$[0.51]$} & {$[0.74]$} & & {$[0.71]$} & {$[0.62]$} & {$[0.84]$} \\
\hline \multirow{3}{*}{$J_{4}$} & 0.58 & 0.50 & 0.71 & \multirow{3}{*}{$J_{10}$} & 0.71 & 0.65 & 0.82 \\
\hline & $\{0.58\}$ & $\{0.49\}$ & $\{0.71\}$ & & $\{0.73\}$ & $\{0.61\}$ & $\{0.78\}$ \\
\hline & {$[0.60]$} & {$[0.51]$} & {$[0.74]$} & & {$[0.72]$} & {$[0.62]$} & [0.85] \\
\hline \multirow{2}{*}{$J_{6}(\mathrm{kNm} / \mathrm{s})$} & 5.35 & 4.33 & 6.83 & \multirow{2}{*}{$J_{12}(\mathrm{kNm} / \mathrm{s})$} & 35.7 & 27.0 & 52.1 \\
\hline & $\{12.0\}$ & $\{8.46\}$ & $\{16.6\}$ & & $\{72.0\}$ & $\{52.7\}$ & $\{118\}$ \\
\hline \multirow{3}{*}{$J_{6}^{*}(\mathrm{kNm} / \mathrm{s})$} & 2.37 & 2.18 & 2.46 & \multirow{3}{*}{$J_{12}^{*}(\mathrm{kNm} / \mathrm{s})$} & 2.33 & 2.24 & 2.82 \\
\hline & $\{2.37\}$ & $\{2.03\}$ & $\{2.62\}$ & & $\{2.34\}$ & $\{2.02\}$ & $\{2.67\}$ \\
\hline & {$[2.38]$} & [2.05] & [2.49] & & {$[2.26]$} & [2.08] & [2.65] \\
\hline \multirow{2}{*}{$\sigma_{u}(\mathrm{kN})$} & 22.7 & 19.2 & 28.3 & \multirow{2}{*}{$\max |u(t)|(\mathrm{kN})$} & 94.2 & 83.1 & 106 \\
\hline & $\{34.1\}$ & $\{28.3\}$ & $\{44.3\}$ & & $\{118\}$ & $\{106\}$ & $\{164\}$ \\
\hline \multirow{3}{*}{$\sigma_{x_{\text {drel }}}(\mathrm{cm})$} & 22.8 & 19.5 & 25.3 & \multirow{3}{*}{$\max \left|x_{\text {drel }}\right|(\mathrm{cm})$} & 76.8 & 70.6 & 93.1 \\
\hline & $\{23.0\}$ & $\{18.4\}$ & $\{27.5\}$ & & $\{74.3\}$ & $\{59.8\}$ & $\{91.6\}$ \\
\hline & {$[22.3]$} & [17.9] & {$[25.0]$} & & {$[69.6]$} & {$[58.0]$} & [84.3] \\
\hline
\end{tabular}

(Figure 3). However, instances of this happening are few, as is evident from the relatively small RMS values of $x_{\text {drel }}$ (given in Table 1) when compared to the linearity limit of $0.55 \mathrm{~m}$ for $\theta=40^{\circ}$. Hence, the model given by (3) is henceforth used in the control law. The stiffness of the device can also be written as

$$
k_{d}(t)=k_{d n}+\Delta k_{d}(t)
$$

Here, $k_{d n}$ is the nominal stiffness that corresponds to the TMD being tuned to the fundamental frequency of the structure. Further, $\Delta k_{d}(t)$ is the additional stiffness required to attain the desired control force. The additional stiffness is obtained by varying the device configuration, $\theta$, such that $\Delta k_{d \text { min }} \leq \Delta k_{d}(t) \leq \Delta k_{d \text { max }}$, where $\Delta k_{d \text { min }}=\left(k_{e} \cos ^{2} \theta_{\text {max }}-\right.$ $\left.k_{d n}\right)$ and $\Delta k_{d \max }=\left(k_{e} \cos ^{2} \theta_{\text {min }}-k_{d n}\right)$.

\section{Reduced Order Model}

The equation of motion of the wind excited building, with SAVS-TMD at the top storey, is written as

$$
\mathbf{M} \ddot{\mathbf{X}}+\mathbf{C X}+\mathbf{K X}=\mathbf{D} u(t)+\mathbf{W}(t) .
$$

Here, $\mathbf{X}=\left[x_{1}, x_{2} \ldots, x_{n-1}, x_{d}\right]^{T}$ is the displacement vector measured relative to the ground, where $x_{i}, i=1, \ldots,(n-1)$, is the lateral displacement of the $i$ th storey and $x_{d}$ is the TMD displacement, $u(t)$ is the control input (i.e., force) that is realized via the additional stiffness provided by the SAVS device, $\mathbf{M}=\operatorname{diag}\left[\mathbf{M}_{s}, m_{d}\right]$ is the system mass matrix, $\mathbf{C}=$ $\operatorname{diag}\left[\mathbf{C}_{s}, \mathbf{0}\right]+\operatorname{diag}\left[\mathbf{0}, \mathbf{C}_{d}\right]$ is the system damping matrix, and $\mathbf{K}=\operatorname{diag}\left[\mathbf{K}_{s}, \mathbf{0}\right]+\operatorname{diag}\left[\mathbf{0}, \mathbf{K}_{d}\right]$ is the system stiffness matrix, where subscript $s$ refers to the structure and subscript $d$ refers to the damper, $\left.\mathbf{K}_{d}=\left[\begin{array}{lll}k_{d n} & -k_{d n}\end{array}\right\}^{T}\left\{\begin{array}{ll}-k_{d n} & k_{d n}\end{array}\right\}^{T}\right]$ and $\left.\mathbf{C}_{d}=\left[\begin{array}{lll}\left\{c_{d}\right. & -c_{d}\end{array}\right\}^{T}\left\{\begin{array}{ll}-c_{d} & c_{d}\end{array}\right\}^{T}\right], m_{d}, c_{d}$, and $k_{d n}$, are the mass, damping coefficient, and nominal stiffness, respectively, of the TMD, $\mathbf{D}=[0,0, \ldots, 1,-1]^{T}$ is the control force placement vector, and $\mathbf{W}(t)$ is the wind excitation vector, with its last element being zero. Since the system stiffness matrix contains only the nominal stiffness of the TMD, the system is timeinvariant and the control force required is given as

$$
u=\Delta k_{d} x_{\mathrm{drel}} ; \quad x_{\mathrm{drel}}=x_{d}-x_{n-1} .
$$

The state space representation of (5) is

$$
\dot{\mathbf{Z}}=\mathbf{A Z}+\mathbf{B} u+\mathbf{E W},
$$

where

$$
\begin{gathered}
\mathbf{Z}=\left[\begin{array}{c}
\mathbf{X} \\
\dot{\mathbf{X}}
\end{array}\right] ; \quad \mathbf{A}=\left[\begin{array}{cc}
\mathbf{0} & \mathbf{I} \\
\mathbf{M}^{-1} \mathbf{K} & \mathbf{M}^{-1} \mathbf{C}
\end{array}\right] ; \\
\mathbf{B}=\left[\begin{array}{c}
\mathbf{0} \\
\mathbf{M}^{-1} \mathbf{D}
\end{array}\right] ; \quad \mathbf{E}=\left[\begin{array}{c}
\mathbf{0} \\
\mathbf{M}^{-1}
\end{array}\right] .
\end{gathered}
$$

The output vector for assessing control effectiveness is given by

$$
\mathbf{y}=\mathbf{C}_{\mathbf{y}} \mathbf{Z}+\mathbf{D}_{\mathbf{y}} u+\mathbf{F W}
$$


where $\mathbf{C}_{\mathbf{y}}, \mathbf{D}_{\mathbf{y}}$, and $\mathbf{F}$ are as defined in Section 5. In order to reduce the computation time, for real-time control applications, the model is reduced by using the method of Davison $[20]$ as done in $[2,14]$. This entails choosing a reduced set of $2 l$ states that are representative of the system response and then expressing this reduced set of states in terms of the first $2 l$ eigenmodes that dominate the response. Let $\Gamma$ represent the matrix of eigenvectors of $\mathbf{A}$, with the eigenvectors arranged in decreasing order of eigenvalue dominance (i.e., the first eigenvector corresponds to the eigenvalue lying closest to the origin and the last eigenvector corresponds to the eigenvalue lying farthest from the origin). Rearrange $\mathbf{Z}$ such that $\mathbf{Z}=$ $\left[\begin{array}{ll}\mathbf{Z}_{r}^{T} & \mathbf{Z}_{o}^{T}\end{array}\right]^{T}$, where $\mathbf{Z}_{r}=\left[\begin{array}{ll}\mathbf{X}_{r}^{T} & \dot{\mathbf{X}}_{r}^{T}\end{array}\right]^{T}$ is the reduced order $2 l$-dimension state vector and $\mathbf{X}_{r}$ is the reduced order $l$ dimension displacement vector. Here, $\mathbf{Z}_{o}$ comprises the 2 ( $n-$ l) states, of the full order system, that are excluded from the reduced order system. The $\mathbf{A}, \mathbf{B}, \mathbf{C}_{\mathbf{y}}, \mathbf{E}, \boldsymbol{\Gamma}$ matrices are now rearranged according to $\mathbf{Z}=\left[\begin{array}{ll}\mathbf{Z}_{r}^{T} & \mathbf{Z}_{o}^{T}\end{array}\right]^{T}$. Thus, the approximation of the states in terms of the first $2 l$ eigenmodes yields [14]

$$
\mathbf{Z}=\left[\begin{array}{ll}
\mathbf{I} & \boldsymbol{\Gamma}_{11}^{-T} \boldsymbol{\Gamma}_{21}^{T}
\end{array}\right]^{T} \mathbf{Z}_{r}
$$

and it also yields the reduced order system

$$
\begin{gathered}
\dot{\mathbf{Z}}_{r}=\mathbf{A}_{r} \mathbf{Z}_{r}+\mathbf{B}_{r} u+\mathbf{E}_{r} \mathbf{W} ; \\
\mathbf{A}_{r}=\mathbf{A}_{11}+\mathbf{A}_{12} \boldsymbol{\Gamma}_{21} \boldsymbol{\Gamma}_{11}^{-1} ; \\
\mathbf{B}_{r}=\boldsymbol{\Gamma}_{11}\left[\mathbf{S}_{1}^{T}, \mathbf{S}_{2}^{T}, \ldots, \mathbf{S}_{2 l}^{T}\right] \mathbf{B} ; \\
\mathbf{E}_{r}=\boldsymbol{\Gamma}_{11}\left[\mathbf{S}_{1}^{T}, \mathbf{S}_{2}^{T}, \ldots, \mathbf{S}_{2 l}^{T}\right] \mathbf{E} ; \\
\mathbf{y}=\mathbf{C}_{r} \mathbf{Z}_{r}+\mathbf{D}_{\mathbf{y}} u+\mathbf{F W} ; \quad \mathbf{C}_{r}=\mathbf{C}_{\mathbf{y}}\left[\begin{array}{ll}
\mathbf{I} & \boldsymbol{\Gamma}_{11}^{-T} \boldsymbol{\Gamma}_{21}^{T}
\end{array}\right]^{T} .
\end{gathered}
$$

Here, $\mathbf{A}_{11}$ is the top left $(2 l \times 2 l)$ partition of $\mathbf{A}, \mathbf{A}_{12}$ is the top right $(2 l \times(2 n-2 l))$ partition of $\mathbf{A}, \Gamma_{11}$ is the top left $(2 l \times 2 l)$ partition of $\Gamma, \Gamma_{21}$ is the bottom left $((2 n-2 l) \times 2 l)$ partition of $\boldsymbol{\Gamma}$, and $\mathbf{S}_{i}$ is the $i$ th row vector of $\boldsymbol{\Gamma}^{-1}$.

\section{Controller Design}

Linear quadratic regulator (LQR) control [21] is considered for controller design. The reduced order model, that is, (11), is considered for the plant dynamics. Thus, the reduced states are assumed to be available for feedback. Using LQR control, the control force $u_{d}$ is obtained such that the performance index

$$
\begin{aligned}
& J=\frac{1}{2} \int_{0}^{\infty}\left[\begin{array}{llll}
\mathbf{Z}^{T} & \mathbf{Q} & \mathbf{Z}+R & u_{d}^{2}(t)
\end{array}\right] \mathrm{d} t \\
& =\frac{1}{2} \int_{0}^{\infty}\left[\begin{array}{llll}
\mathbf{Z}_{r}^{T} & \mathbf{Q}_{r} & \mathbf{Z}_{r}+R & u_{d}^{2}
\end{array}\right] \mathrm{d} t
\end{aligned}
$$

is minimized. The performance index represents the total energy of the system (i.e., the structure and the SAVS-TMD). Here, $R$ is the positive scalar weighting of the control effort, and $\mathbf{Q}$ is the $(2 n \times 2 n)$ positive semidefinite state weighting matrix; that is,

$$
\mathbf{Q}=\operatorname{diag}\left[\begin{array}{llll}
\mathbf{K}_{\mathbf{s}} & \beta_{1} & \mathbf{M}_{\mathbf{s}} & \beta_{2}
\end{array}\right] .
$$

Here, $\beta_{1}, \beta_{2}$, and $R$, are chosen such that effective control is achieved without exceeding the limits prescribed on the TMD displacement, $x_{\text {drel }}$, and the device configuration angle $\theta$. Equations $(10)$ and $(14)$ yield the $(2 l \times 2 l)$ positive semidefinite state weighting matrix for the reduced order system as

$$
\mathbf{Q}_{r}=\left[\mathbf{I}\left[\boldsymbol{\Gamma}_{21} \boldsymbol{\Gamma}_{11}^{-1}\right]^{T}\right] \mathbf{Q}\left[\mathbf{I}\left[\boldsymbol{\Gamma}_{21} \boldsymbol{\Gamma}_{11}^{-1}\right]^{T}\right]^{T} .
$$

Here, $\mathbf{Q}$ has been rearranged according to $\mathbf{Z}=\left[\begin{array}{ll}\mathbf{Z}_{r}^{T} & \mathbf{Z}_{o}^{T}\end{array}\right]^{T}$. Minimizing $J$ subject to the constraint represented by (11) considered without wind excitation, one obtains the desired optimal control force as [21]

$$
u_{d}=-R^{-1} \mathbf{B}_{r}^{T} \mathbf{P Z} \mathbf{r}_{\mathbf{r}}
$$

Here, $\mathbf{P}$ is the solution of the algebraic Riccati equation given as

$$
\mathbf{P A}_{r}+\mathbf{A}_{r}^{T} \mathbf{P}-\mathbf{P B}_{r} R^{-1} \mathbf{B}_{r}^{T} \mathbf{P}+\mathbf{Q}_{r}=\mathbf{0} .
$$

Considering (4), (6), and the configuration limits of the SAVS device, one obtains the control law. This yields the position of joint-1, that is required to realize the desired control force $u_{d}$, as

$$
\theta=\left\{\begin{array}{cl}
41^{\circ} \text { (open position) } & \text { if } \frac{u_{d}}{x_{\mathrm{drel}}} \leq \Delta k_{d \text { min }} \\
\cos ^{-1} \sqrt{\frac{k_{d n}+u_{d} / x_{\mathrm{drel}}}{k_{e}}} & \text { if } \Delta k_{d \min }<\frac{u_{d}}{x_{\mathrm{drel}}}<\Delta k_{d \max } \\
7^{\circ} \text { (closed position) } & \text { if } \frac{u_{d}}{x_{\mathrm{drel}}} \geq \Delta k_{d \max }, \\
q_{1}=L_{e} \sin \theta .
\end{array}\right.
$$

Equations (3) and (4) yield the control force thus realized; that is,

$$
u=\left(k_{e} \cos ^{2} \theta(t)-k_{d n}\right) x_{\mathrm{drel}}
$$

An alternative procedure to determine the device configuration $q_{1}$ that involves the solution of the nonlinear static equations (1) is as follows. For a known desired control force, $u_{d}$, and TMD displacement, $x_{\text {drel }}$, the total force required from the TMD is obtained as $F_{d}=k_{d n} x_{\text {drel }}+u_{d}$. Using $F_{d}$ and $x_{\text {drel }}$ in (1), the device actuation, $q_{1}$, as well as $q_{2}$, $q_{3}$, and $q_{4}$, can be obtained, subject to the minimum values permissible for $\theta_{1}$ and $\theta_{4}$. In this manner, the linear forcedisplacement relation, (3), and the resulting control law, (19), are not used. However, as discussed in Section 2 (on the basis of RMS values of the TMD displacement), the linear forcedisplacement relation suffices for the present application. Hence, this alternative procedure is not adopted.

\section{Results}

The 76-storey benchmark building is modeled using 76 translational degrees of freedom, as considered in [2]. The TMD, having mass $m_{d}=500 t$, is placed at the top storey. 

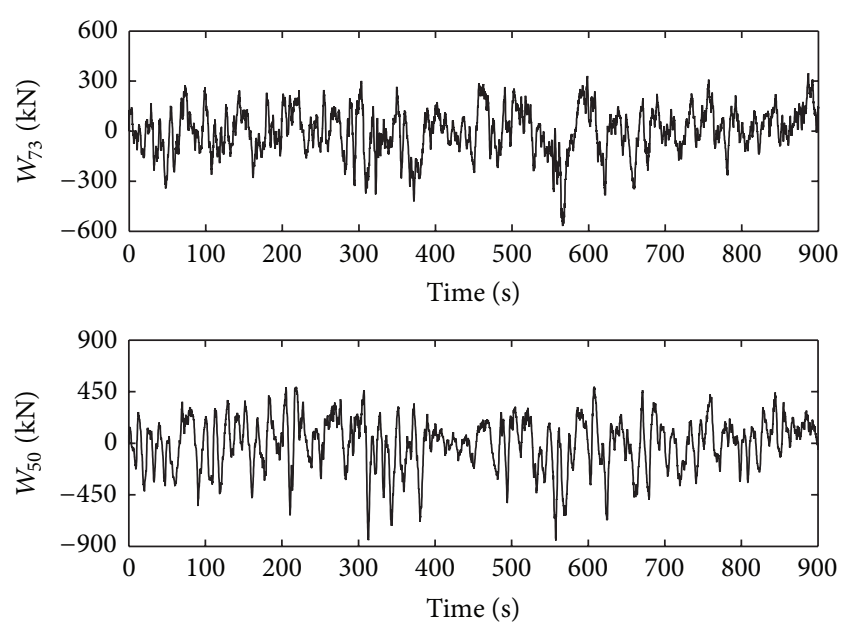

FIGURE 4: Time history of wind force on the 50th and 73rd storeys [22].

The mass matrix, $\mathbf{M}_{s}$, stiffness matrix, $\mathbf{K}_{s}$, and damping matrix $\mathbf{C}_{s}$ for the structure, as well as the across-wind data, are considered from [22]. A $\pm 15 \%$ variation in structural stiffness is also considered in order to assess the effectiveness of the controller [2]. The damping ratio for the TMD is considered as $7 \%[12]$.

As one of the aims of this study is to compare results from STLC with those using ALC [2] and STSC [13], the reduced order model of [2] is considered. This is a 24-degreeof-freedom model, with the reduced state, $\mathbf{X}_{r}$, comprising the displacements at storeys $3,6,10,13,16,20,23,26,30$, $33,36,40,43,46,50,53,56,60,63,66,70,73$, and 76 , and the TMD displacement, all measured relative to ground. The wind force vector is obtained by lumping wind forces at the reduced DOFs, with the wind location matrix $\mathbf{E}_{r}$ modified appropriately [2]. Thus, the third part of (12) is not considered when obtaining the wind force vector. Figure 4 shows the resulting across-wind load that acts on storeys 50 and 73 for the reduced order model.

The nominal stiffness of the SAVS device, $k_{d n}$, is tuned to the fundamental frequency of the structure. The fundamental frequency is $0.16 \mathrm{~Hz}$, resulting in $k_{d n}=505.12 \mathrm{kN} / \mathrm{m}$. Using (3), the stiffness of the spring used in the device is chosen as $k_{e}=650 \mathrm{kN}$. This ensures that the nominal stiffness of the device is the average of the stiffness values of the device at its operational limits $\theta_{\min }=7^{\circ}$ and $\theta_{\max }=41^{\circ}$. Thus, the limits on the additional stiffness that can be provided by the device are $\Delta k_{d \max }=135.223$ and $\Delta k_{d \text { min }}=-134.89 \mathrm{kN} / \mathrm{m}$.

The parameters for the controller design are chosen as $\beta_{1}=900, \beta_{2}=1$, and $R=2 \times 10^{-2}$. The reduced order plant, that is, (11), is integrated using MATLAB ode-45. The initial conditions for the state and the initial control input are considered as zero (i.e., $\mathbf{Z}_{r}=\mathbf{0}$ and $u=0$ ). Thus, $\mathbf{Z}_{r}$ is obtained at the end of each time step. Subsequently, the desired control force, $u_{d}$, is obtained from (17), the position of joint-1, $q_{1}$, is obtained from the control law, that is, (19), and the control force, $u$, applied at the start of the next time step is obtained from (20). Figure 5 shows the block diagram for the control loop simulation.

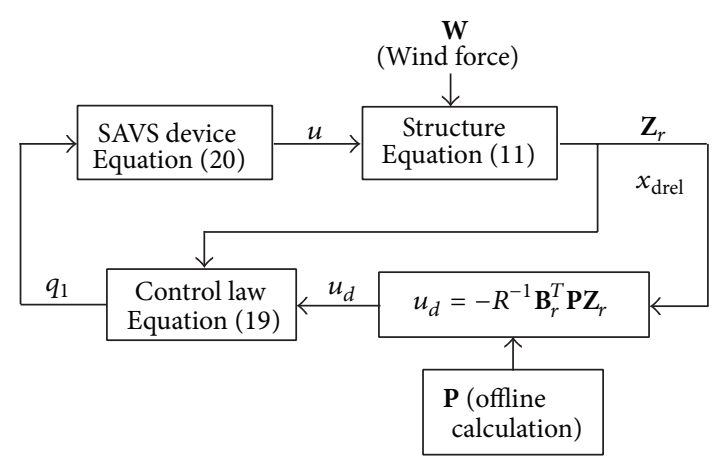

FIGURE 5: Block diagram for control.

Performance criteria denoted by $J_{1}, \ldots, J_{12}$ are evaluated [2]. These are defined in terms of controlled responses that are suitably normalized wherever indicated. The uncontrolled structure with zero variation in stiffness is considered when obtaining the normalizing quantity which, unless noted otherwise, corresponds to the response being normalized. The displacement and acceleration of storeys 1, 30, 50, 55, $60,65,70,75$, and 76 and the displacement and velocity of the TMD measured relative to storey 76 are considered. Hence, the matrices $\mathbf{C}_{\mathbf{y}}, \mathbf{D}_{\mathbf{y}}$, and $\mathbf{F}$, appearing in the output equation, that is, (13), are defined as follows. Define $\mathbf{v}=\left[\begin{array}{lllllllll}1 & 30 & 50 & 55 & 60 & 65 & 70 & 75 & 76\end{array}\right]^{T}$. For $i=1$ to $9, \mathbf{C}_{\mathbf{y}}(i, \mathbf{v}(i))=1$. Further, $\mathbf{C}_{\mathbf{y}}(10,77)=-\mathbf{C}_{\mathbf{y}}(10,76)=$ $\mathbf{C}_{\mathbf{y}}(11,154)=-\mathbf{C}_{\mathbf{y}}(11,153)=1$. For $i=12$ to 20 , the $i$ th row of $\mathbf{C}_{\mathbf{y}}, \mathbf{D}_{\mathbf{y}}$, and $\mathbf{F}$ is the $(77+\mathbf{v}(i-11))$ th row of $\mathbf{A}, \mathbf{B}$, and $\mathbf{E}$, respectively. Here, $\mathbf{v}(i-11)$ denotes the $(i-11)$ th element of $\mathbf{v}$. The remaining elements of $\mathbf{C}_{\mathbf{y}}, \mathbf{D}_{\mathbf{y}}$, and $\mathbf{F}$ are zero.

$J_{1}$ denotes the maximum RMS acceleration, normalized with the RMS acceleration of storey 75 , with storey 76 and the TMD being excluded from the maximum. $J_{2}$ denotes the average of the normalized RMS accelerations, with the average being taken over storeys 50 to 75 . $J_{3}$ denotes the normalized RMS displacement of storey $76 . J_{4}$ denotes the average of the normalized RMS displacements, with the average being taken over storeys 50 to 76 . $J_{5}$ denotes the RMS of $x_{\text {drel }}$ (i.e., the TMD displacement relative to storey 76) normalized with the RMS displacement of storey $76 ; J_{6}$ denotes the RMS of $u \dot{x}_{\text {drel }}$, that is, the average input power. Note that in [13] the "average power" is defined as the RMS of $\dot{x}_{\text {drel }}$ normalized with the RMS velocity of storey 76 , that is, a ratio of velocities. This is denoted here as $\widehat{J}_{6}$ and is used for comparison with the results from STSC. This essentially represents the RMS of the TMD velocity. Performance criteria $J_{7}$ to $J_{12}$ and $\widehat{J}_{12}$ are defined analogous to $J_{1}$ to $J_{6}$ and $\widehat{J}_{6}$, respectively, by replacing the RMS values with corresponding peak values. The constraints stipulated in the benchmark problem are $\sigma_{u} \leq 100 \mathrm{kN}$ for the RMS control force, $\sigma_{x_{\text {drel }}} \leq$ $30 \mathrm{~cm}$ for the RMS TMD-displacement, $\max |u| \leq 300 \mathrm{kN}$ for the peak control force, and $\max \left|x_{\text {drel }}\right| \leq 95 \mathrm{~cm}$ for the peak TMD-displacement.

The present STLC results are compared with the ALC results and the passive TMD results obtained by Yang et al. [2, 22] for $20 \%$ damping ratio and with the STSC results obtained 

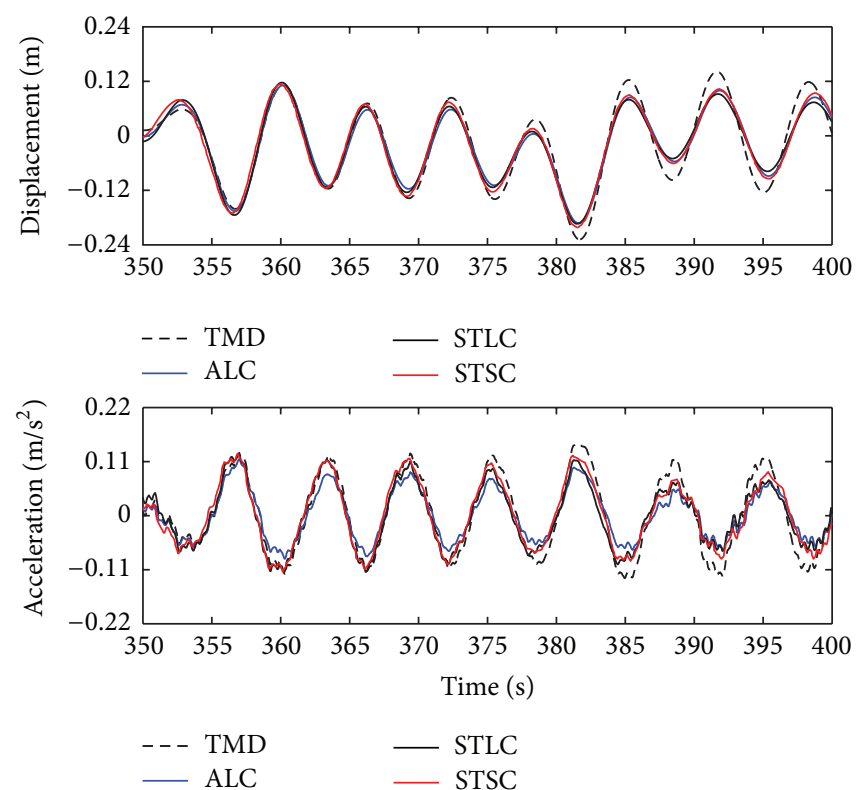

FIGURE 6: Comparison of the 75th storey Response (0\% stiffness variation).

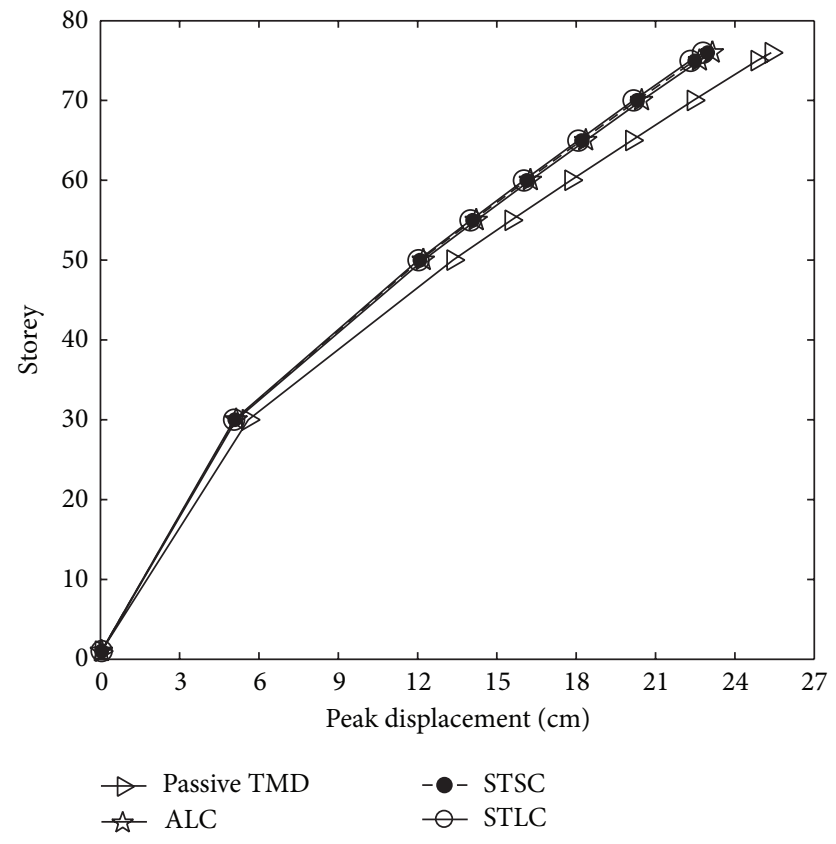

(a)

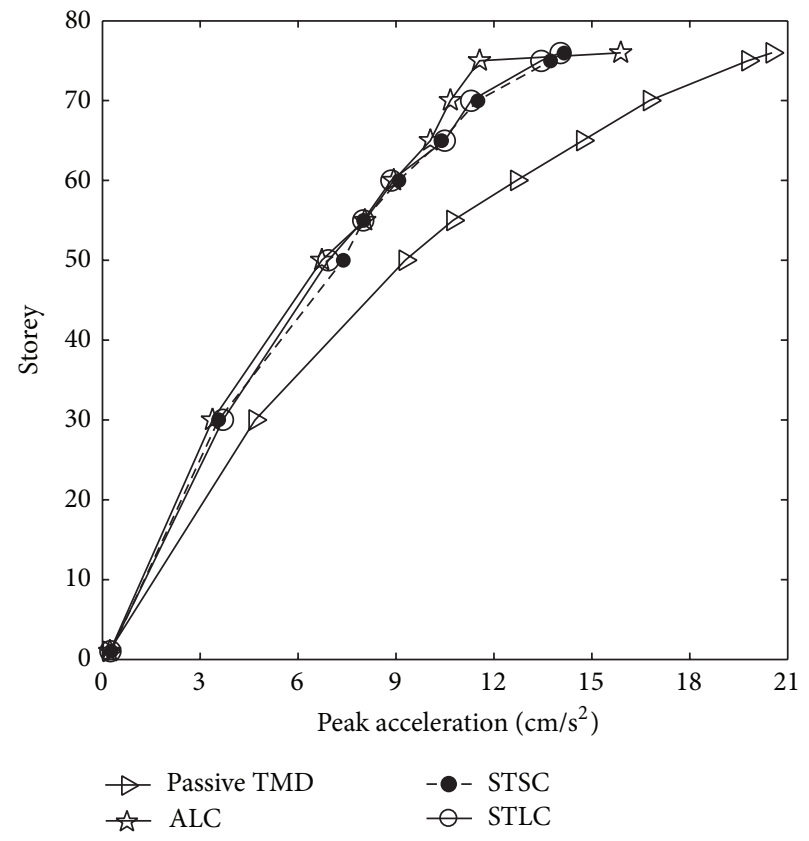

(b)

Figure 7: Comparison of peak displacement and peak acceleration ( $0 \%$ stiffness variation).

by Nagarajaiah and Varadarajan [13] for 7\% damping ratio. Figure 6 compares the time histories of displacement and acceleration of storey 75 . The case of $0 \%$ stiffness variation is considered. The present STLC yields displacements that are comparable with those from ALC and STSC. The acceleration control obtained from STLC is marginally better than that from STSC, but it is somewhat inferior when compared to ALC results. The passive TMD is the least effective of the three controllers.
Peak responses from the four controllers, for the case of $0 \%$ stiffness variation, are compared in Figure 7 . The passive TMD reduces peak displacements by $17-21 \%$ as compared to the uncontrolled structure. The STLC, STSC, and ALC methods yield a further reduction of $7-10 \%$ in peak displacements, as compared to the passive-TMD controlled structure. Peak displacements from STLC, STSC, and ALC are comparable, with the present STLC being marginally lower than ALC and STSC. The present STLC yields a reduction of $21-32 \%$ in 


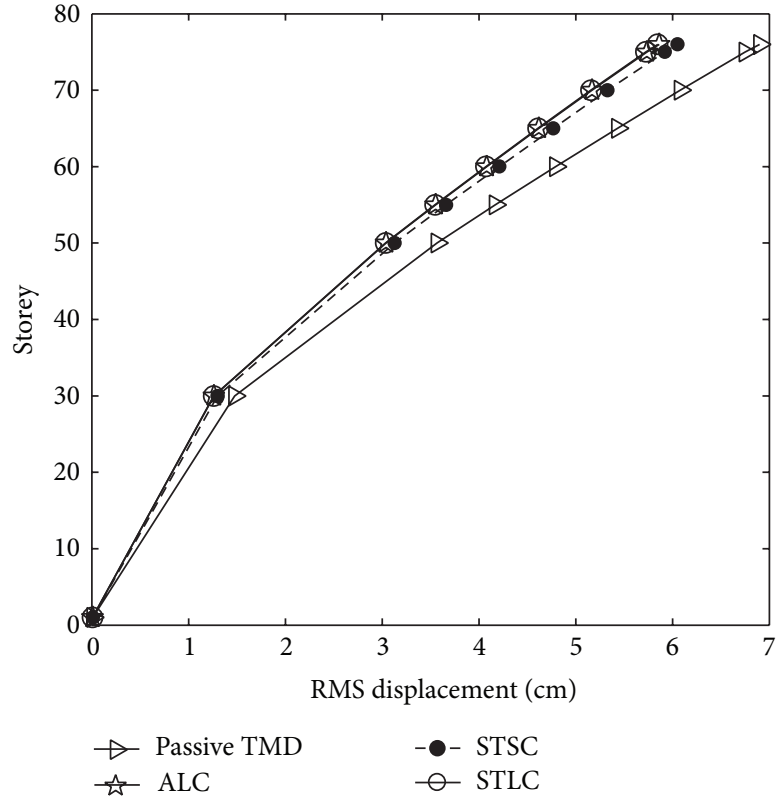

(a)

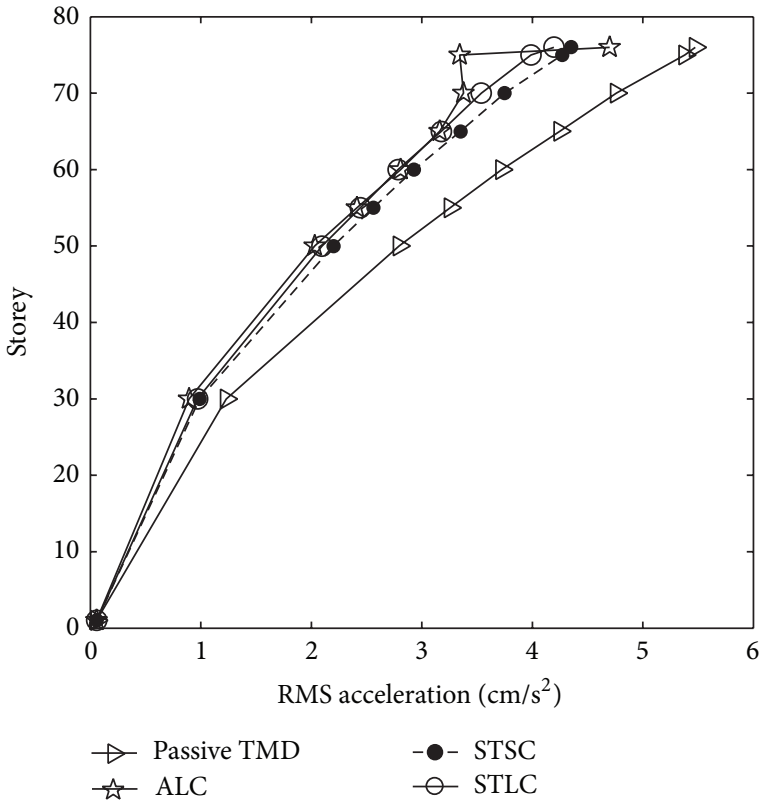

(b)

FIGURE 8: Comparison of RMS displacement and RMS acceleration ( $0 \%$ stiffness variation).

peak accelerations when compared to passive TMD control. It yields marginally lower peak accelerations compared to STSC. However, STLC mostly yields an increase of up to $10 \%$ in peak accelerations when compared to ALC. Thus, for $0 \%$ stiffness variation, STLC provides storeywise peak responses that are comparable with results of STSC and ALC, except in respect of accelerations for which STLC is inferior as compared to ALC. Note that ALC is generally better than STLC or STSC in acceleration control, since it is an active method whereby the desired control force can always be attained (up to a $300 \mathrm{kN}$ limit) by the actuator and is independent of the ATMD displacement. In contrast, the force attained by the SAVS-TMD at a particular instant is limited by the range of device stiffness (i.e., $505 \pm 135 \mathrm{kN} / \mathrm{m}$ ) and the TMD displacement at that instant.

RMS responses from the four controllers, for the case of $0 \%$ stiffness variation, are compared in Figure 8 . The passive TMD yields up to $32 \%$ reduction in displacements and up to $42 \%$ reduction in accelerations, as compared to the uncontrolled structure. The present STLC attenuates displacements by $15 \%$ and accelerations by $21-26 \%$, as compared to passive TMD control. It yields a marginal $3 \%$ reduction in displacements and up to $7 \%$ reduction in acceleration, as compared to STSC. It is comparable in displacements but yields accelerations that are mostly higher, by up to $9 \%$, when compared to ALC. Thus, for $0 \%$ stiffness variation, STLC provides comparable-to-moderately-better storeywise RMS responses as compared to STSC and ALC, except for RMS accelerations for which it is inferior as compared to ALC.

Peak and RMS responses from the four controllers, for the case of $+15 \%$ stiffness variation, are compared in
Figures 9 and 10, respectively. When compared to passiveTMD control, the present STLC yields an increase of up to $4 \%$ in peak displacements, a reduction of $8-14 \%$ in peak accelerations, a reduction of around 5\% in RMS displacements, and a reduction of 4-10\% in RMS accelerations. When compared to STSC, STLC yields an increase of up to $5 \%$ in peak displacements and $9 \%$ in peak accelerations. When compared to ALC, STLC yields an increase of up to $6 \%$ in peak displacements, $8-25 \%$ in peak accelerations, up to $3 \%$ in RMS displacements, and 6-22\% in RMS accelerations. Thus, for $+15 \%$ stiffness variation, STLC provides mostly inferior storeywise peak and RMS responses as compared to STSC and ALC.

Peak and RMS responses from the four controllers, for the case of $-15 \%$ stiffness variation, are compared in Figures 11 and 12, respectively. When compared to passive-TMD control, the present STLC yields a reduction of around 19\% in peak displacements, $14-25 \%$ in peak accelerations, $13 \%$ in RMS displacements, and $18-24 \%$ in RMS accelerations. When compared to STSC, STLC yields a reduction of around $4 \%$ in peak displacements and up to $8 \%$ in peak accelerations. When compared to ALC, STLC yields an increase of around $5 \%$ in peak displacements, but a marginal reduction in RMS displacements. The peak and RMS accelerations show a mixed trend, that is, the performance of STLC ranges from a reduction of $5 \%$ to an increase of $12 \%$ in peak accelerations, and a reduction of $12 \%$ to an increase of $20 \%$ in RMS accelerations. Thus, for $-15 \%$ stiffness variation, STLC provides storeywise displacement responses comparable to results from STSC and ALC and moderately better storeywise peak accelerations as compared to STSC and a mixed trend in storeywise peak and RMS accelerations vis-a-vis ALC. 


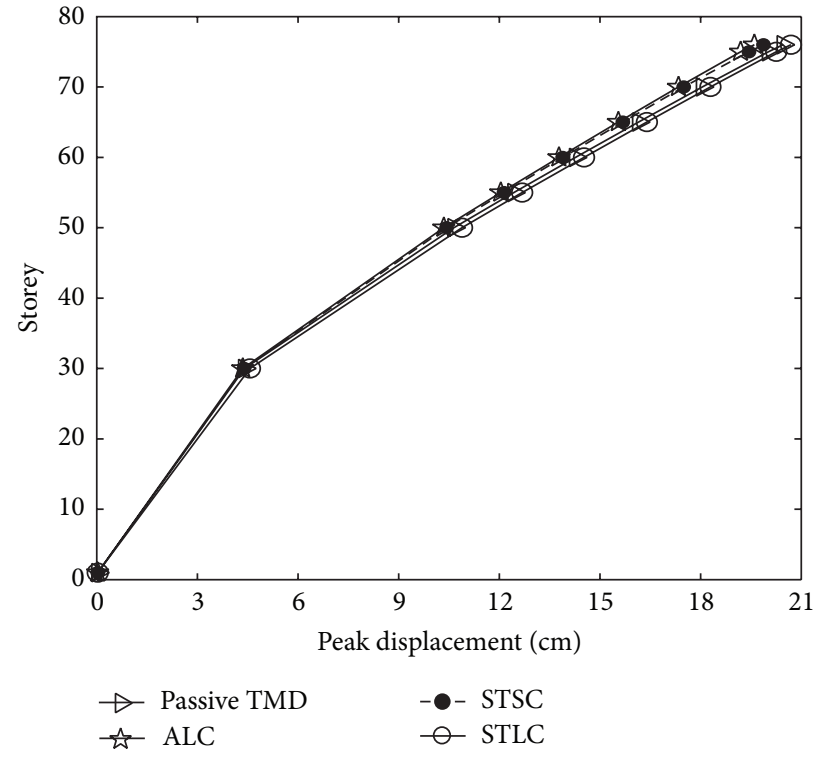

(a)

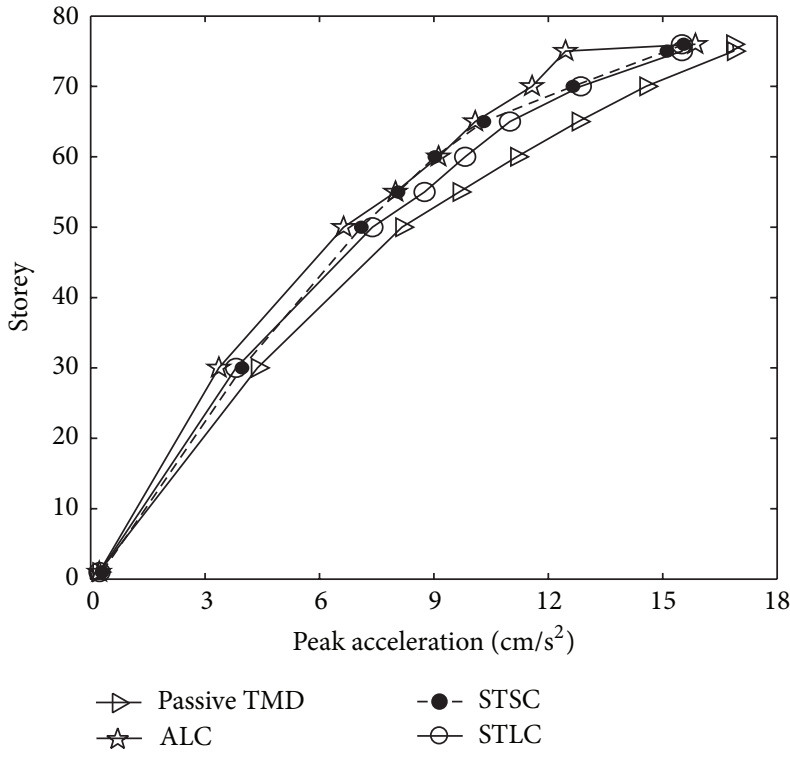

(b)

FIGURE 9: Comparison of peak displacement and peak acceleration (15\% stiffness variation).

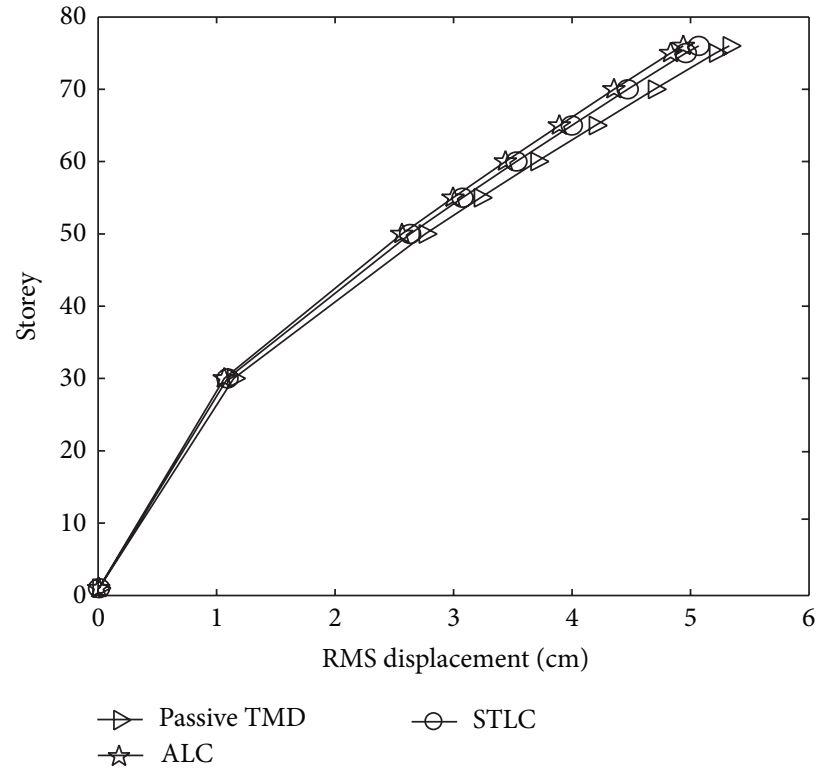

(a)

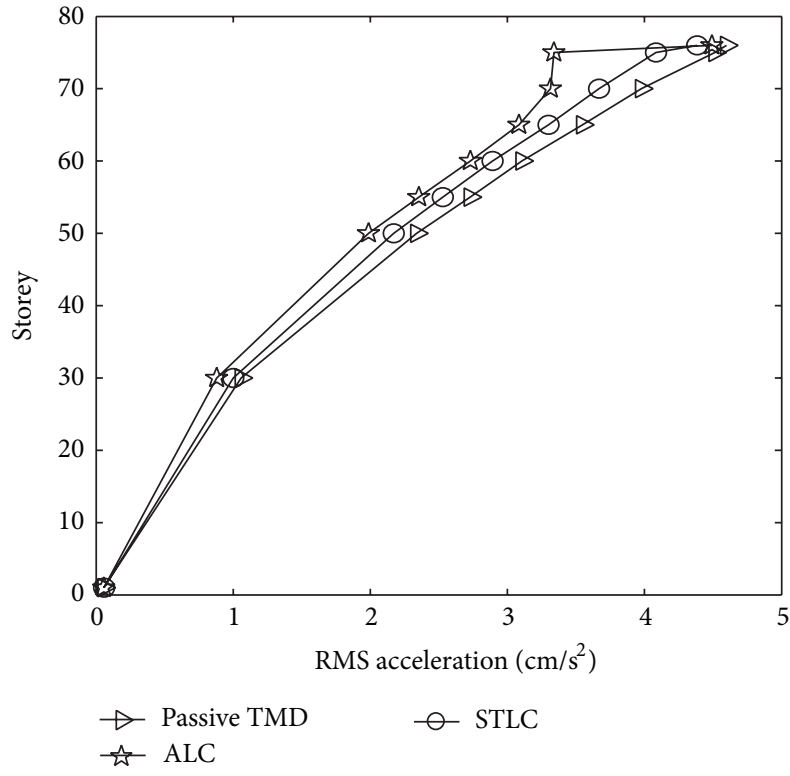

(b)

FIGURE 10: Comparison of RMS displacement and RMS acceleration (15\% stiffness variation).

Performance criteria $J_{1}-J_{12}$ obtained using the present STLC are compared with those from ALC (shown in parentheses) and STSC (shown in square brackets) in Table 1. STLC yields RMS and peak displacements, that is, $J_{3}, J_{4}, J_{9}$, $J_{10}$, that are comparable with results from ALC and STSC. The average RMS acceleration, $J_{2}$, obtained from STLC is comparable with results from ALC for the $0 \%$ and $-15 \%$ stiffness variation cases, $10 \%$ higher than the ALC results for the $15 \%$ stiffness variation case, comparable with results from STSC for the $0 \%$ and $15 \%$ stiffness variation cases, and 9\% lower than the STSC results for the $-15 \%$ stiffness variation case. The maximum RMS acceleration, $J_{1}$, obtained from STLC is $18 \%, 22 \%$, and $16 \%$ higher than the ALC results for the $0 \%,+15 \%$, and $-15 \%$ stiffness variation cases, respectively, comparable with results from STSC for the 15\% stiffness variation case, and 7\% and 10\% lower than the STSC results for the $0 \%$ and $-15 \%$ stiffness variations, respectively. The average peak acceleration, $J_{8}$, obtained from STLC is comparable with results from ALC for the $0 \%$ and $-15 \%$ stiffness variation cases, $12 \%$ higher than the ALC results for 


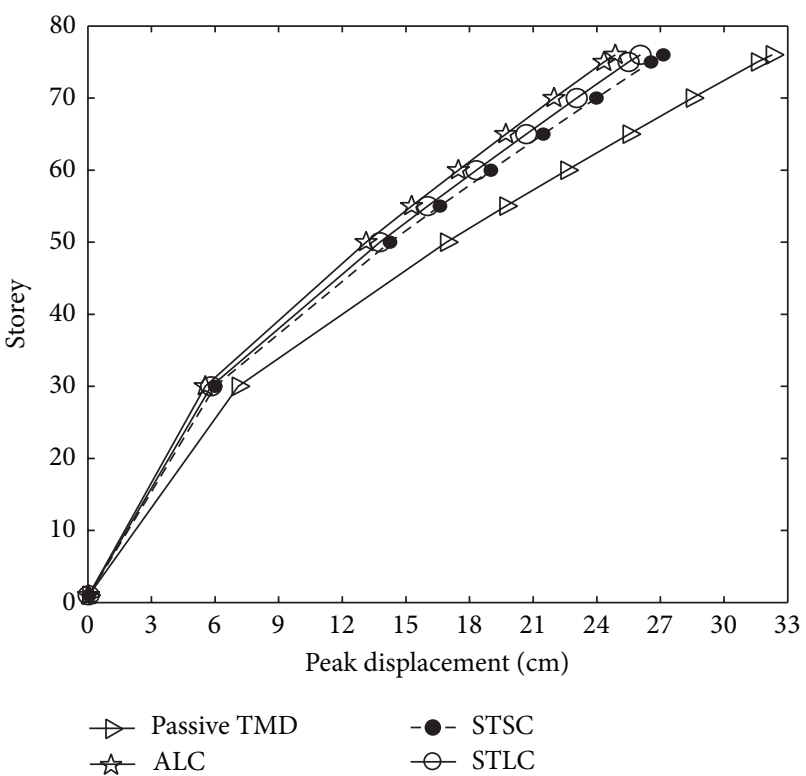

(a)

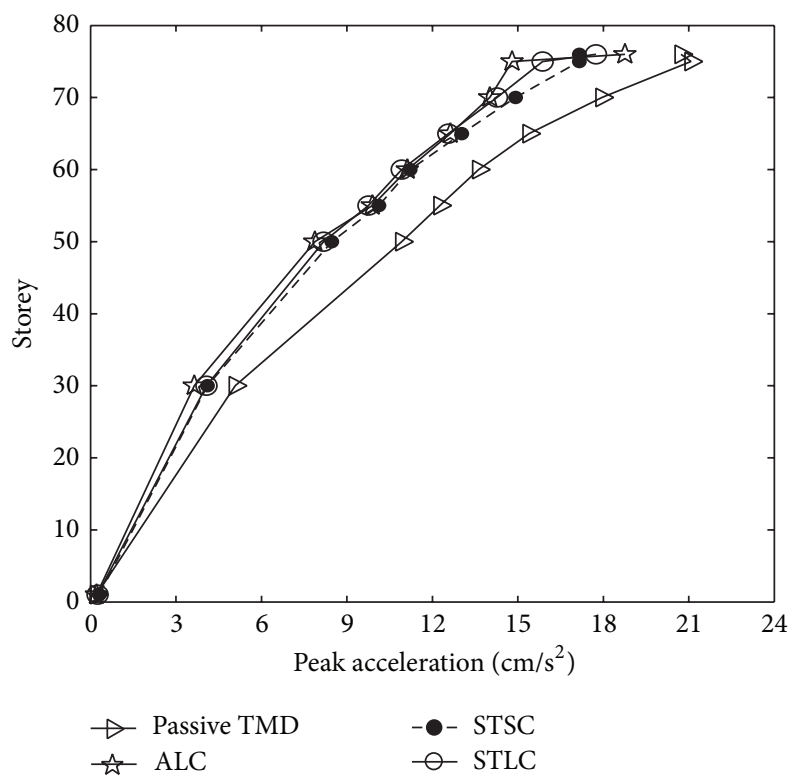

(b)

FIGURE 11: Comparison of peak displacement and peak acceleration ( $-15 \%$ stiffness variation).

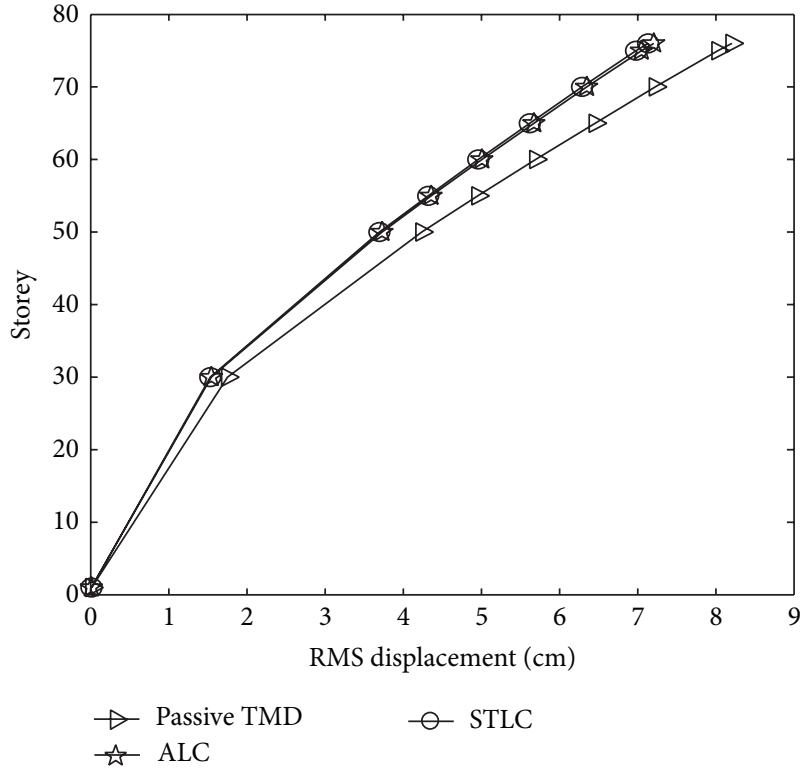

(a)

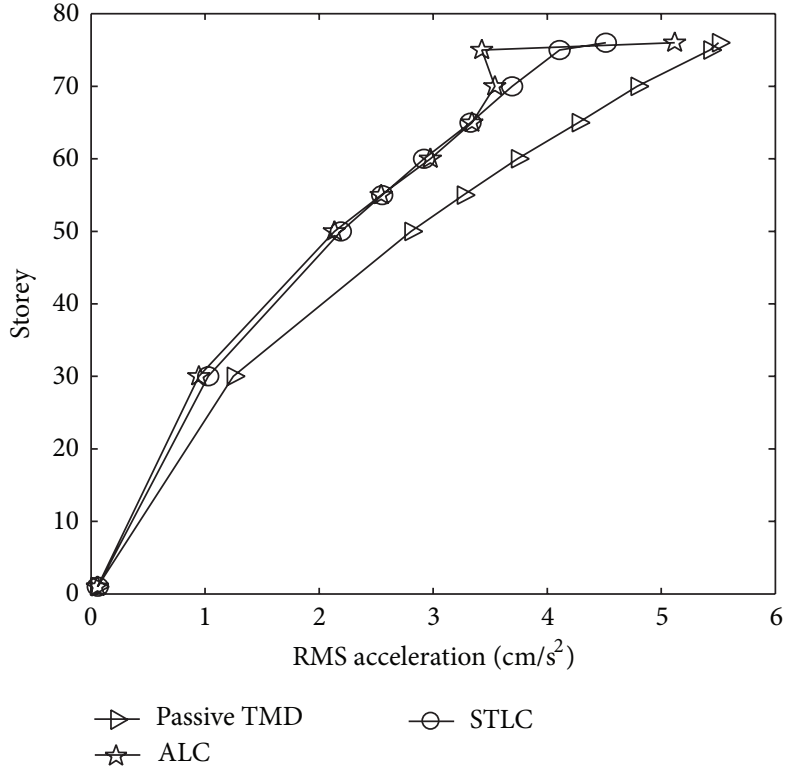

(b)

FIGURE 12: Comparison of RMS displacement and RMS acceleration (-15\% stiffness variation).

the $15 \%$ stiffness variation case, comparable with results from STSC for all three stiffness variation cases. The maximum peak acceleration, $J_{7}$, obtained from STLC is $16 \%, 24 \%$, and $7 \%$ higher than the ALC results for the $0 \%,+15 \%$, and $-15 \%$ stiffness variation cases, respectively, comparable with results from STSC for the $0 \%$ and $15 \%$ stiffness variation cases and $8 \%$ lower than the STSC results for the $-15 \%$ stiffness variation case.
Thus, STLC provides displacement control that is comparable to that provided by ALC/STSC. However, the acceleration control obtained from STLC is not as good as that obtained from ALC; that is, the average-peak and averageRMS accelerations for the $+15 \%$ stiffness variation case and the max-peak and max-RMS accelerations for all three stiffness variation cases show lesser attenuation when using STLC as compared to ALC. The reason for this, as discussed 
previously, is that ALC is an active control method that yields superior acceleration control. The acceleration control obtained from STLC is better than that obtained from STSC for the $-15 \%$ stiffness variation case, and otherwise comparable with the STSC results.

The RMS relative displacement of the SAVS-TMD, that is, $\sigma_{x_{\text {drel }}}$ obtained from STLC is $6 \%$ higher than the ALC result for the $+15 \%$ stiffness variation case, $8 \%$ lower than the ALC result for the $-15 \%$ stiffness variation case, comparable with results from ALC for the $0 \%$ stiffness variation case, and $9 \%$ higher than the STSC results for the 15\% stiffness variation case, comparable with results from STSC for the $0 \%$ and $-15 \%$ stiffness variation cases. Note that, for ALC, the actuator stroke is considered instead of the SAVS-TMD displacement. The RMS displacement of storey 76 is $10.14 \mathrm{~cm}$ for the $0 \%$ stiffness variation case. Since $J_{5}$ is obtained by merely normalizing $\sigma_{x_{\text {drel }}}$ with this value, it is omitted from Table 1. The peak relative displacement of the SAVS-TMD, that is, $\max \left|x_{\text {drel }}\right|$, is the highest when using STLC, that is, higher by as much as $18 \%$ and $22 \%$ vis-a-vis ALC and STSC, respectively. The peak displacement of storey 76 is $32.30 \mathrm{~cm}$ for the $0 \%$ stiffness variation case. Since $J_{11}$ is obtained by merely normalizing max $\left|x_{\text {drel }}\right|$ with this value, it is omitted from Table 1.

The average power expended, $J_{6}$, and the peak power expended, $J_{12}$, when using STLC are at least $49 \%$ lower visa-vis ALC, since the latter is a fully active control method. The RMS control force, $\sigma_{u}$, is at least $32 \%$ lower, and the peak control force, $\max |u(t)|$, is at least $21 \%$ lower, when using STLC vis-a-vis ALC. Thus, the semiactive STLC requires substantially less power and control force in order to achieve comparable displacement control and acceptable acceleration control vis-a-vis ALC. Note that the RMS and the peak values of both the SAVS-TMD displacement and the control force provided by the SAVS-TMD are within limits prescribed by the benchmark problem. Comparisons between STLC and STSC-for power and control force-are excluded, since the values of $J_{6}, J_{12}, \sigma_{u}$, and $\max |u(t)|$ are not available for STSC. The index $\widehat{J}_{6}$ obtained from STLC varies between $6 \%$ lower and $7 \%$ higher than the ALC results, and it is up to $6 \%$ higher than the STSC results. The index $\widehat{J}_{12}$ obtained from STLC is up to $11 \%$ higher than the ALC results and up to $8 \%$ higher than the STSC results. However, as noted previously, $\widehat{J}_{6}$ and $\widehat{J}_{12}$ represent the RMS and peak values, respectively, of the TMD velocity; that is, they do not actually represent the power input.

Figure 13 shows the time history of the applied and the desired control forces for the $0 \%$ stiffness variation case. The applied force depends on the relative displacement and the stiffness of the SAVS-TMD. Since the device stiffness available is limited, due to the constraints imposed on the configuration angle, the semiactive device is not always able to produce the desired force. The RMS value of the difference between applied and desired forces is $38.6 \mathrm{kN}$. Figure 14 shows the time history of the device position, that is, $q_{1}$ (Figure 2). The horizontal portions represent the limits on the device configuration (19). The device position, and hence its stiffness, varies smoothly except when there is a change in

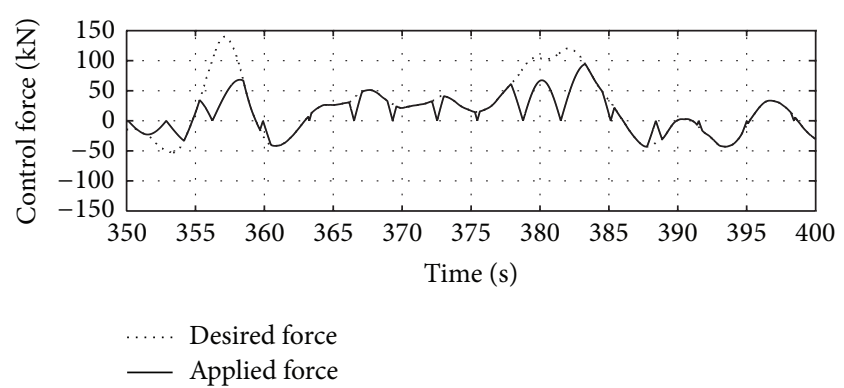

FIGURE 13: Time history of desired and applied control force $(0 \%$ stiffness variation).

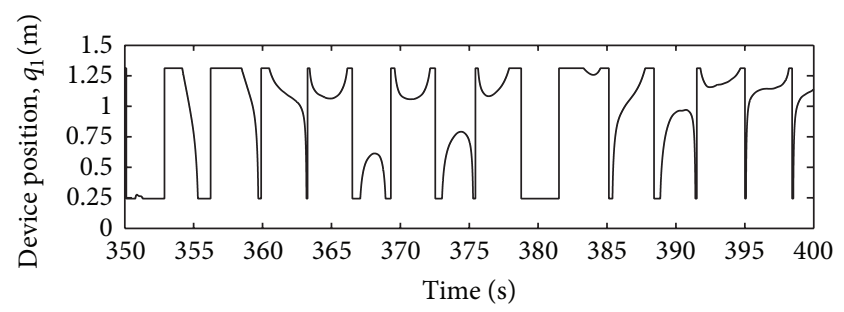

FIgURE 14: Time history of device position ( $0 \%$ stiffness variation).

the direction of its relative displacement $x_{\text {drel }}(19)$. When the device position changes abruptly, the applied control force is very small due to $x_{\text {drel }}$ being small at these instants. However, the applied control force does not change abruptly at these instants.

\section{Conclusion}

LQR control is employed for a wind excited benchmark building that is fitted with a semiactive variable stiffness TMD device. The nominal stiffness of the device is tuned to the fundamental frequency of the structure and included in the system stiffness matrix. The additional, time-varying, component of the device stiffness is obtained via LQR control and a suitable control law and utilized to apply the control force. A nonlinear static analysis is done to establish the operational limits on the device configuration angle, so as to ensure near-linear behavior of the device and thus yield a simple control law.

Comparison of the present STLC with ALC and STSC permits the following conclusions.

(i) The performance criteria show that STLC generally provides displacement control that is comparable with that of ALC and STSC. However, the acceleration control from STLC is not as good when compared with ALC results. The acceleration control from STLC is better than that from STSC for the $-15 \%$ stiffness variation case, and otherwise comparable with STSC results.

(ii) For the 0\% stiffness variation case, STLC provides comparable-to-moderately-better peak and RMS storeywise responses, except that, for accelerations, it is inferior vis-a-vis ALC (since the latter is 
a fully active method). For the $+15 \%$ stiffness variation case, STLC generally provides inferior peak and RMS storeywise responses. For the $-15 \%$ stiffness variation case, STLC provides storeywise displacement responses comparable with ALC and STSC results, moderately better peak storeywise accelerations vis-a-vis STSC, and a mixed trend in peak and RMS storeywise accelerations vis-a-vis ALC.

(iii) The STLC requires substantially less power and control force to achieve comparable displacement control and acceptable acceleration control vis-a-vis ALC.

Future work would involve LQG and Static Output Feedback controller designs that reduce the number of sensors required. Further, knowing the SAVS-TMD displacement (i.e., $\left.x_{\text {drel }}\right)$ and the desired control force (i.e., $F_{d}$, obtained by linear control methods), one can obtain the device actuation required (i.e., $q_{1}$ ) via the nonlinear static equation (1) or their dynamic counterparts (in case the masses of the sliders are substantial). This would eliminate the limiting of the device configuration angle and would thus reduce the error between desired and applied control forces.

\section{Conflict of Interests}

The authors declare that there is no conflict of interests regarding the publication of this paper.

\section{References}

[1] F. Ricciardelli, A. D. Pizzimenti, and M. Mattei, "Passive and active mass damper control of the response of tall buildings to wind gustiness," Engineering Structures, vol. 25, no. 9, pp. 11991209, 2003.

[2] J. N. Yang, A. K. Agrawal, B. Samali, and J.-C. Wu, "Benchmark problem for response control of wind-excited tall buildings," ASCE Journal of Engineering Mechanics, vol. 130, no. 4, pp. 437446, 2004.

[3] G. W. Housner, L. A. Bergman, T. K. Caughey et al., "Structural control: past, present, and future," ASCE Journal of Engineering Mechanics, vol. 123, no. 9, pp. 897-971, 1997.

[4] M. D. Symans and M. C. Constantinou, "Semi-active control systems for seismic protection of structures: a state-of-the-art review," Engineering Structures, vol. 21, no. 6, pp. 469-487, 1999.

[5] T. Kobori, M. Takahashi, T. Nasu, N. Niwa, and K. Ogasawara, "Seismic response controlled structure with active variable stiffness system," Earthquake Engineering and Structural Dynamics, vol. 22, no. 11, pp. 925-941, 1993.

[6] T. Nasu, T. Kobori, M. Takahashi, N. Niwa, and K. Ogasawara, "Active variable stiffness system with non-resonant control," Earthquake Engineering and Structural Dynamics, vol. 30, no. 11, pp. 1597-1614, 2001.

[7] D. C. Nemir, Y. Lin, and R. A. Osegueda, "Semiactive motion control using variable stiffness," ASCE Journal of Structural Engineering, vol. 120, no. 4, pp. 1291-1306, 1994.

[8] J. N. Yang, J. C. Wu, and Z. Li, "Control of seismic-excited buildings using active variable stiffness systems," Engineering Structures, vol. 18, no. 8, pp. 589-596, 1996.
[9] J. N. Yang, J.-H. Kim, and A. K. Agrawal, "Resetting semiactive stiffness damper for seismic response control," ASCE Journal of Structural Engineering, vol. 126, no. 12, pp. 1427-1433, 2000.

[10] S. Nagarajaiah, "Structural vibration damper with continuously variable stiffness," U.S. Patent 6098969, 2000.

[11] S. Nagarajaiah and E. Sonmez, "Structures with semiactive variable stiffness single/multiple tuned mass dampers," ASCE Journal of Structural Engineering, vol. 133, no. 1, pp. 67-77, 2007.

[12] N. Varadarajan and S. Nagarajaiah, "Wind response control of building with variable stiffness tuned mass damper using empirical mode decomposition/Hilbert transform," ASCE Journal of Engineering Mechanics, vol. 130, no. 4, pp. 451-458, 2004.

[13] S. Nagarajaiah and N. Varadarajan, "Short time Fourier transform algorithm for wind response control of buildings with variable stiffness TMD," Engineering Structures, vol. 27, no. 3, pp. 431-441, 2005.

[14] J. C. Wu and J. N. Yang, "Active control of transmission tower under stochastic wind," ASCE Journal of Structural Engineering, vol. 124, no. 11, pp. 1302-1312, 1998.

[15] R. Collins, B. Basu, and B. M. Broderick, "Bang-bang and semiactive control with variable stiffness TMDs," ASCE Journal of Structural Engineering, vol. 134, no. 2, pp. 310-317, 2008.

[16] K.-V. Yuen, Y. Shi, J. L. Beck, and H.-F. Lam, "Structural protection using MR dampers with clipped robust reliabilitybased control," Structural and Multidisciplinary Optimization, vol. 34, no. 5, pp. 431-443, 2007.

[17] A. K-Karamodin and H. H-Kazemi, "Semi-active control of structures using neuro-predictive algorithm for MR dampers," Structural Control and Health Monitoring, vol. 17, no. 3, pp. 237253, 2010.

[18] H.-C. Sohn, K.-T. Hong, K.-S. Hong, and W.-S. Yoo, "An adaptive LQG control for semi-active suspension systems," International Journal of Vehicle Design, vol. 34, no. 4, pp. 309326, 2004.

[19] L. Gaul, H. Albrecht, and J. Wirnitzer, "Semi-active friction damping of large space truss structures," Shock and Vibration, vol. 11, no. 3-4, pp. 173-186, 2004.

[20] E. J. Davison, "A method for simplifying linear dynamic systems," IEEE Transactions on Automatic Control, vol. 11, no. 1, pp. 93-101, 1966.

[21] D. S. Naidu, Optimal Control Systems, CRC Press, New York, NY, USA, 2003.

[22] http://sstl.cee.illinois.edu/benchmarks. 

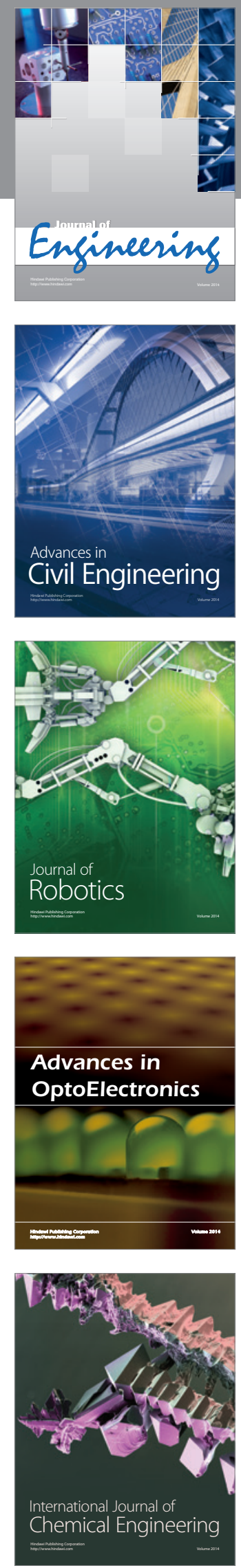

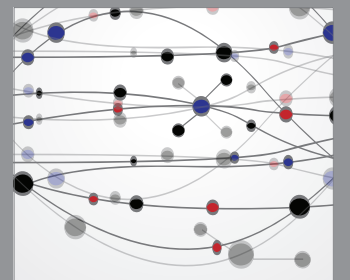

The Scientific World Journal
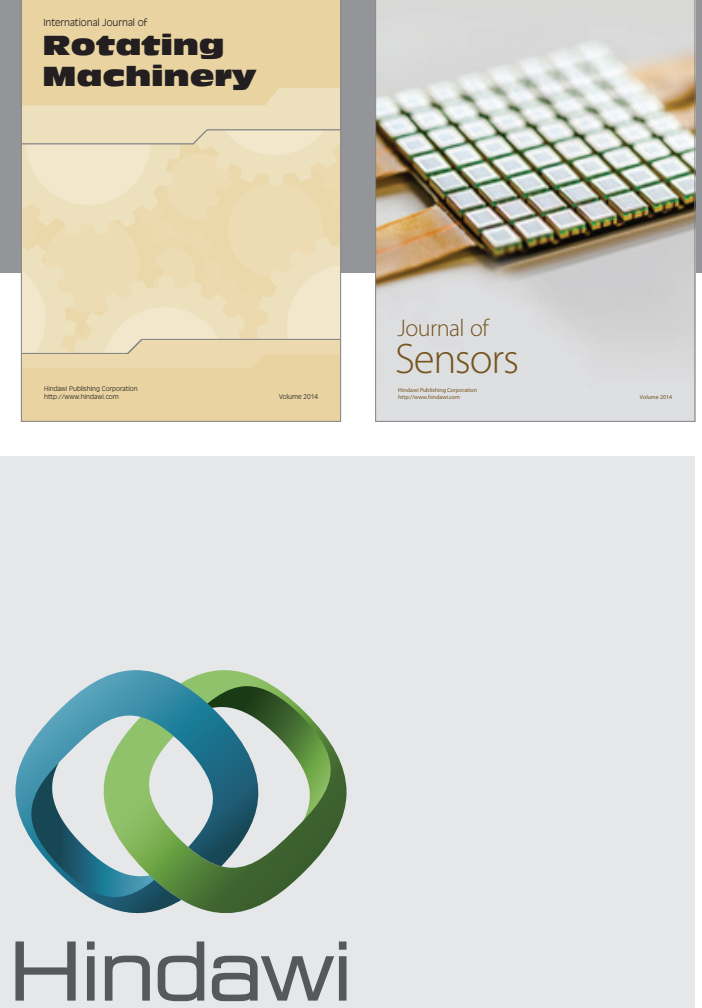

Submit your manuscripts at http://www.hindawi.com
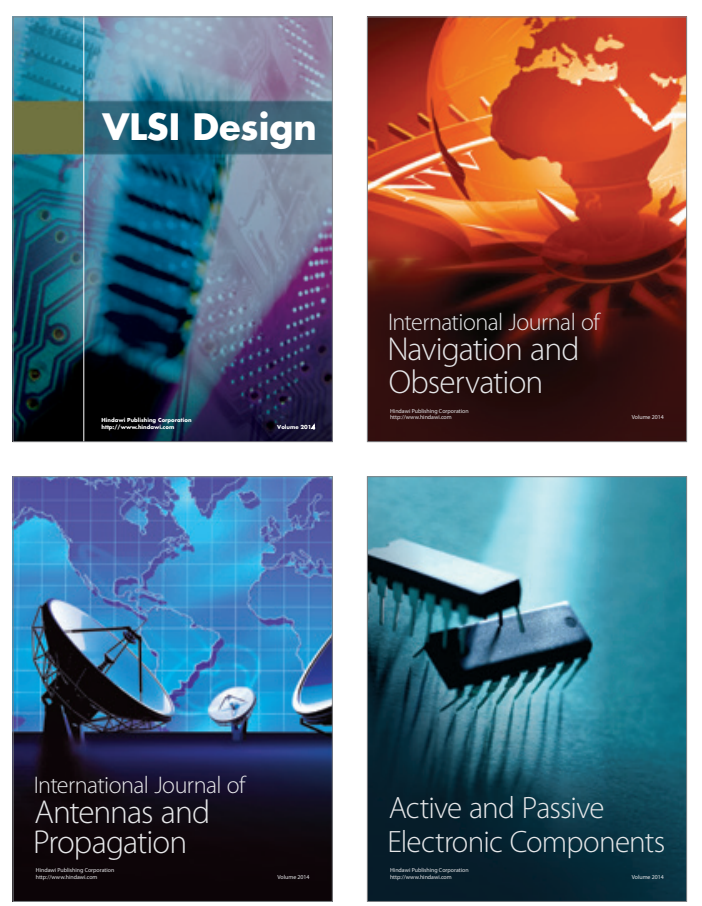
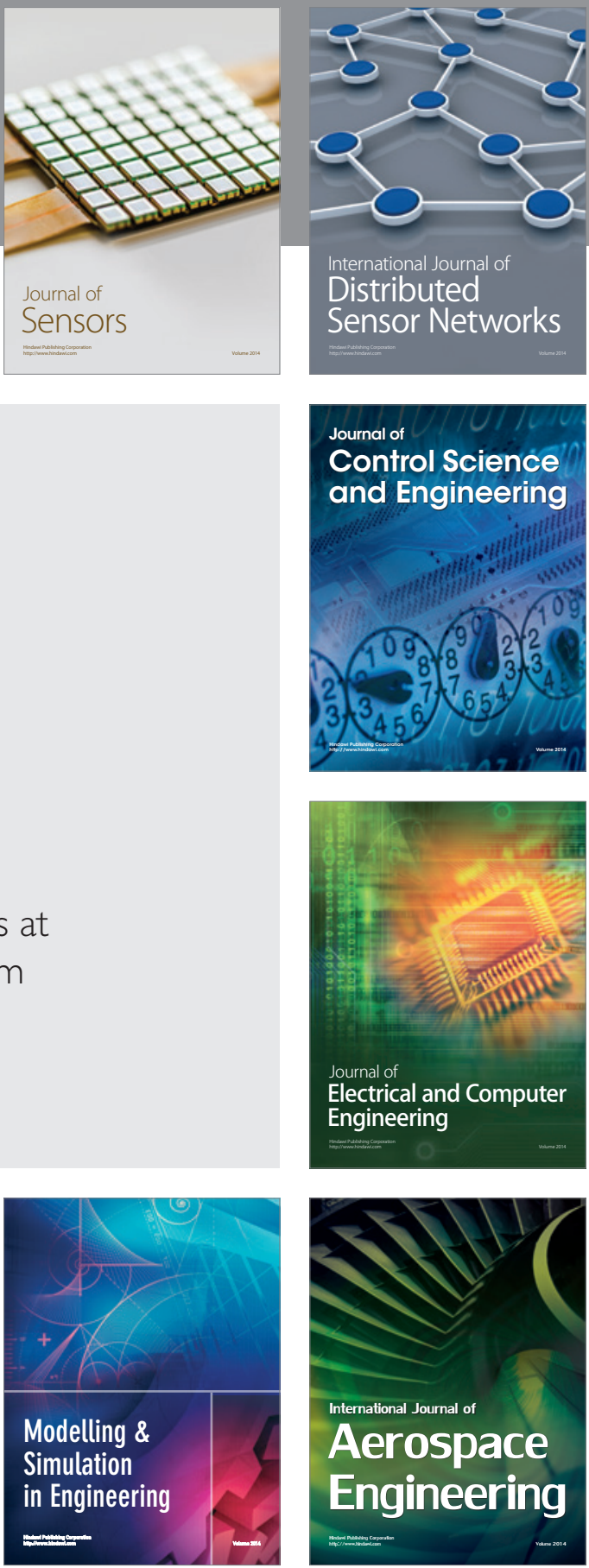

Journal of

Control Science

and Engineering
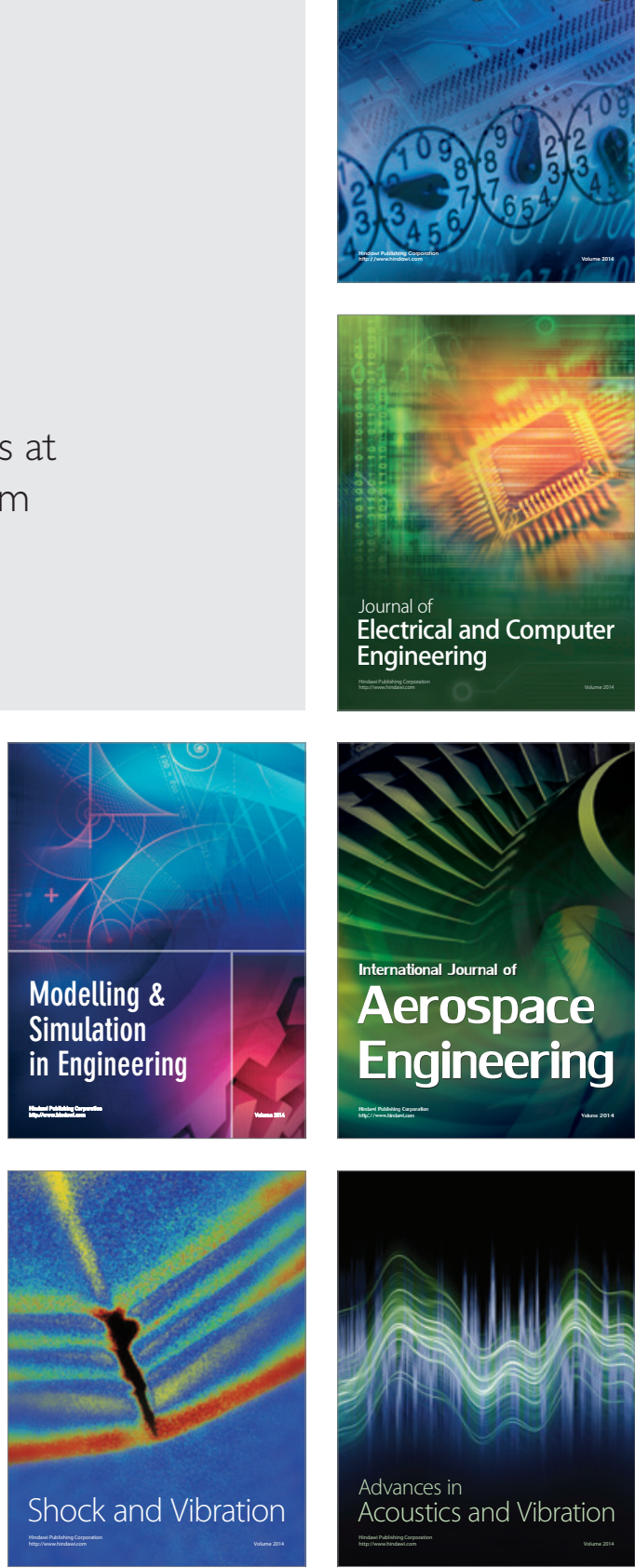